\title{
Auslandsaufenthalt als Kompetenzschule? - Eine empirische Untersuchung der Auswirkungen von Auslandsaufenthalten auf die berufsbezogenen Kompetenzbereiche
}

\author{
Petia Genkova ${ }^{1}$ (D) Lisa Kruse ${ }^{1}$ \\ Angenommen: 2. Januar 2021 / Online publiziert: 15. Januar 2021 \\ () Der/die Autor(en) 2021
}

\section{Zusammenfassung}

Der vorliegende Beitrag zur Zeitschrift Gruppe. Interaktion. Organisation. (GIO) zeigt, basierend auf einer Onlinestudie, dass Auslandsaufenthalte mit höheren arbeitsbezogenen Kompetenzen assoziiert sind. In diesem Beitrag wird also analysiert, inwieweit bei Auslandsaufenthalten von einer Kompetenzschule die Rede sein kann. Auslandserfahrung ist ein fester Bestandteil vieler Curricula geworden. Auslandsaufenthalten wird nicht nur eine Verbesserung von Fremdsprachkenntnissen zugesprochen, sondern auch die Förderung allgemeinerer Kompetenzen sowie eine Weiterentwicklung der Persönlichkeit. Insbesondere wird vermutet, dass Umgang mit Stress und Ambivalenzen, Erfahrungen im Projektmanagement, interkulturelle und soziale Kompetenzen durch die Auslandserfahrungen zunehmen. In der vorliegenden Studie wurde untersucht, inwieweit ein Auslandsaufenthalt, diesen Erwartungen gerecht wird, ob also Kompetenzunterschiede zwischen Personen, die einen Auslandsaufenthalt absolvierten und jenen, die keinen absolvierten, sichtbar sind. Es konnte bestätigt werden, dass Personen mit Auslandserfahrung tatsächlich höhere Werte in den Dimensionen Arbeitsengagement, beruflicher Ehrgeiz, Widerstandskraft, Distanzierungsfähigkeit, offensive Problembewältigung, Engagement, soziale Kompetenz, Dominanz sowie kulturelle Intelligenz aufweisen. Kulturelle Intelligenz hängt sowohl mit der Länge der Auslandsaufenthalte als auch mit der Qualität der Kontakte zu Angehörigen der Gastkultur zusammen. Die Ergebnisse werden diskutiert und abschließend Implikationen für weitere Forschung und die Praxis abgeleitet.

Schlüsselwörter Auslandsaufenthalt · Kulturelle Intelligenz · Soziale Kompetenz · Kompetenzschule · Arbeitsengagement $\cdot$ Resilienz

Prof. Dr. phil. habil. Petia Genkova

petia@genkova.de

Lisa Kruse, M.A.

lisa-kruse@freenet.de

1 University of Applied Sciences Osnabrück, Caprivistraße 30a, 49076 Osnabrück, Deutschland 


\title{
Can we consider stays abroad as competence schools? The relationship of stays abroad with job related competences
}

\begin{abstract}
The present article, published in "Gruppe. Interaktion. Organisation. (GIO)", demonstrates, based on an online study, that stays abroad are associated with higher job-related skills and competences. Thus, this article analyzes to the extent one can call stays abroad a competence school. Experience abroad has become an integral part of many curricula. Stays abroad are not only expected to improve foreign language skills, but also to promote more general competences and contribute to the development of personality. In particular, it is assumed, that coping with stress and ambiguity, experience in project management as well as intercultural and social competences are increased by experiences abroad. In the present study, it was examined to what extent a stay abroad meets these expectations, i.e. whether differences in skills are visible between people who did a stay abroad and those who did not. actually show higher values in the dimensions of work commitment, professional ambition, resilience, ability to distance themselves, active problem solving, engagement, social competence, dominance and cultural intelligence. Moreover, interestingly, cultural intelligence correlates with both the length of stays abroad and the quality of local contacts during this period. The results are discussed and implications for further research and practice derived.
\end{abstract}

Keywords Stay abroad $\cdot$ Cultural intelligence $\cdot$ Social competence $\cdot$ Competence school $\cdot$ Engagement $\cdot$ Resilience

\section{Auslandsaufenthalt als Kompetenzschule? - Eine empirische Untersuchung der Auswirkungen von Auslandsaufenthalten auf die berufsbezogenen Kompetenzbereiche}

Mit der zunehmenden Globalisierung der Weltwirtschaft geht notwendigerweise eine verstärkte internationale Ausrichtung von Unternehmen einher. Um unter zunehmend komplexen Umständen Leistungsfähigkeit zu gewährleisten, wird von Angestellten Flexibilität verlangt (Biemann 2009). Hochschulen und Universitäten reagieren bereits auf die wachsende internationale Verflechtung der Wirtschaft und die damit verbundenen Anforderungen. Nachwuchskräften wird deshalb gezielt internationale Mobilität ermöglicht. Zentrale Elemente sind hierbei der Bologna-Prozess (Bologna-Erklärung 1999) und die internationale Ausrichtung der Studiengänge (Heublein et al. 2008). Die europäische Kommission (2010) fördert diese Entwicklungen und erkennt die Mobilität von Studierenden als wichtiges Werkzeug der Ausbildung von Kompetenzen an, um auf dem nationalen und internationalen Arbeitsmarkt erfolgreich zu sein. Diese Annahmen über den Nutzen von Auslandsaufenthalten spiegeln sich ebenfalls in den Ergebnissen von Arbeitgeberbefragungen wider. Auslandsaufenthalte stellen daher auf dem Arbeitsmarkt eine wichtige Zusatzqualifikation dar und bilden somit eine geeignete Strategie, sich von seinen Mitbewerberenden abzuheben (Biemann 2009).

Immer mehr Studien untersuchen die individuellen Entwicklungen verschiedener Kompetenzen durch Auslandserfahrungen. Dabei stehen vor allem die Persönlichkeitsentwicklung und der Umgang mit kulturellen Gegebenheiten im Fokus (Hammer 2015; Schartner 2016; Repo et al.
2017). Der Zusammenhang zwischen Auslandserfahrungen und berufsbezogenen Kompetenzen sowie dem späteren $\mathrm{Ar}$ beitsleben ist jedoch noch immer unzureichend erforscht. Auch die Langzeiteffekte hinsichtlich der Persönlichkeitsentwicklung sowie die Bandbreite der erworbenen Kompetenzen sind wenig untersucht. Die vorliegende Studie wurde mit dem Ziel erhoben, die Zusammenhänge von Auslandsaufenthalten mit den berufsbezogenen Kompetenzbereichen Arbeitsengagement, Widerstandskraft gegen Stress (,Resilienz"), kulturelle Intelligenz, Stabilität, soziale Kompetenz, Kooperationsbereitschaft und Dominanz zu beleuchten, die in Forschung und Praxis als gute Prädiktoren beruflicher Leistungsfähigkeit etabliert sind (Kanning 2019). Mit anderen Worten: Die vorliegende Studie untersucht die Fragestellung, ob ein Auslandsaufenthalt als Kompetenzschule angesehen werden kann. Um diese Frage zu beantworten, werden Querschnittsvergleiche zwischen Personen mit und ohne Auslandsaufenthalt hinsichtlich ihrer beruflichen Kompetenzen vorgenommen. Dadurch trägt diese Studie dazu bei, den berufsbezogenen Kompetenzgewinn im Rahmen eines Auslandsaufenthaltes besser zu verstehen und für die praxisorientierte Kompetenzforschung zugänglich zu machen.

\section{Theoretischer Hintergrund}

\subsection{Anforderungen der Arbeitswelt}

Aufgrund der stetig steigenden Anforderungen in Bezug auf Anpassungsfähigkeit an neue Informationen und Kommunikationssysteme, soziale Dynamik und an die globale Wettbewerbsfähigkeit (Scherrmann 2015) sind Arbeit- 
Tab. 1 Akkulturationsvarianten. (Nach Dick et al. 2014, S. 4)

\begin{tabular}{llll}
\hline & \multicolumn{2}{l}{ Wird es als wertvoll erachtet, die eigene kulturelle Identität und die kulturellen Bräuche und } \\
Wird es als wertvoll betrachtet, & - & „Ja“ & "Nein“ \\
$\begin{array}{llll}\text { Beziehungen zu anderen Gruppen zu } \\
\text { unterhalten? }\end{array}$ & „Ja“ & Integration & Assimilation \\
& „Nein“ & Segregation/Separation & Marginalisierung \\
\hline
\end{tabular}

nehmer*innen zunehmenden psychischen Belastungen und Beanspruchungen ausgesetzt. Es wird in der Praxis vermutet, dass die Resilienz zur Alltagsbewältigung bei Personen mit Auslandserfahrung eine höhere Ausprägung aufweisen (Scherrmann 2015). Als Ursache für die höhere Resilienz werden Erfahrungen mit der Bewältigung von kulturellen Stresssituationen und Ambiguitäten in Betracht gezogen. Besonders das Leben und Arbeiten in einem unbekannten kulturellen Umfeld bedeutet eine Verschärfung der allgemeinen Stresssituation. Trotz der zunehmenden Globalisierung (und der damit einhergehenden allmählichen Herausbildung einer globalen Kultur) stellt ein längerer Aufenthalt in einer fremden Kultur immer noch eine große Herausforderung dar (Genkova und Ringeisen 2015, 2017). Dass der intensive Kontakt mit fremden Kulturen einen Stressfaktor darstellt, ist in der Literatur unumstritten (Helfrich 2013; Thomas 2003). Ob die vermutete erhöhte Resilienz eine Auswirkung auf die Gesundheit hat, wird in dieser Studie aufgrund des limitierten Umfangs nicht untersucht.

\subsection{Akkulturation und Akkulturativer Stress}

Die Sozialisation in einer Kultur ist ein natürlicher Vorgang, der gemeinsame Werte, Wahrnehmungs- und Verhaltensmuster schafft (Helfrich 2013; Thomas 2003). Dadurch erwerben die Mitglieder sowohl ein Orientierungssystem für das eigene Handeln, wie auch einen Maßstab für die Frage, wer zur Gesellschaft gehört und wer nicht. Als Folge unterschiedlicher Wertesysteme unterscheiden sich auch die Wahrnehmung und Interpretation innerer und äußerer Reize zwischen Individuen unterschiedlicher Kulturen.

Trotz der damit verbundenen Schwierigkeiten wandern schon seit langem viele Menschen aus ihrer Heimatkultur aus, mit verschiedenen Graden der Freiwilligkeit. Migration von Individuen wird definiert als die dauerhafte Verlagerung des Lebensmittelpunktes an einen anderen Ort, unabhängig davon, ob dies freiwillig oder unfreiwillig geschieht (Thomas 2003). In der heutigen globalisierten Zeit stehen vor allem Austauschschüler*innen, Auslandsstudierende und Arbeitende in ausländischen Tochterunternehmen im Fokus der Erforschung kurzfristiger Migration (Ward et al. 2001). Für diese Menschen ist Wanderung in vielen Fällen zu einem normalen, alltäglichen Prozess geworden, bei dem man sich regelmäßig an neue Gegebenheiten der sozialen Umwelt anpassen muss. Jedoch geht diese Anpassung oft nicht reibungslos vonstattengeht, sodass Individuen in diesem Fall vor der Entscheidung stehen, den Auslandsaufenthalt abzubrechen oder sich der neuen Umgebung unter Kompromissen anzupassen (Zick 2010).

Akkulturation beschreibt dabei die Art und Weise, wie Menschen ihre kulturellen Muster als Folge von stetigem, direktem Kontakt mit einer neuen Kultur ändern. Unter Akkulturationseinstellungen sind nach Berry (1990) solche Erwartungen $\mathrm{zu}$ verstehen, die Gruppenangehörige eines kulturellen Musters an das Zusammenleben mit Angehörigen einer Gruppe mit einem davon abweichenden kulturellen Muster haben. Hierbei handelt es sich um Präferenzen bezüglich des Anpassungsgrades an die neuen Kulturmuster und bezüglich der Häufigkeit des direkten Kontaktes zu Angehörigen der Fremdgruppe (Berry 1990). Aus der Beantwortung dieser beiden Fragen leiten sie vier Akkulturationsvarianten ab (siehe Tab. 1), die sich im Falle der Migration sowohl auf die Gastgeber-Kultur, als auch auf die Fremdgruppe, beziehungsweise deren einzelne Mitglieder innerhalb der neuen Kultur anwenden lassen.

Überwiegen die Präferenzen, die für einen integrativen Akkulturationsstil typisch sind, so wird die Entstehung einer multikulturellen Gesellschaft begünstigt. Hierbei behalten beide Gruppen ihre kulturellen Muster überwiegend bei, bewegen sich jedoch auch auf die jeweils andere Kultur zu. So entstehen geteilte Muster, Werte und Normen (Dick et al. 2014).

Jede Akkulturation ist mit der Herausforderung verbunden, sich den neuen Bedingungen anzupassen, woraus der sogenannte Akkulturationsstress entsteht. Akkulturationsstress wird somit definiert als der Stress von Mitgliedern der Minderheitsgesellschaft, welcher mit der herkunftsbezogenen Ablehnung durch das soziale Umfeld verbunden ist. Darunter werden alle Faktoren gefasst, die das Gelingen oder Misslingen der Integration bedingen. Relevante Faktoren nennen Silbereisen und Schmitt-Rodermund (1993) mit der Beherrschung der Landessprache, Netzwerksicht, freiwilliger oder unfreiwilliger Migration sowie den Erwartungen an das neue Land.

\subsection{Arbeitsengagement}

Der psychische Druck steigt für Arbeitnehmer*innen, nicht nur durch interkulturell bedingten Stress, sondern auch durch den technischen Fortschritt und der Forderung der Arbeitgeber nach beruflichem Ehrgeiz, Flexibilität, Perfektionismus und Verausgabungsbereitschaft. Schaarschmidt 
und Fischer (2008) ordnen die genannten Faktoren in ihrer Studie dem Arbeitsengagement zu. Für den Arbeitgeber ist dieses ein entscheidender Faktor bei der Einstellung neuer Bewerber*innen. Arbeitsengagement wird durch überdurchschnittliche Abschlussnoten, verschiedene Arbeitszeugnisse und Zusatzqualifikationen im Auswahlprozess eingeschätzt (Schaarschmidt und Fischer 2008; Kanning 2019). Ein Auslandsaufenthalt kann in diesem Zusammenhang eine geeignete Zusatzqualifikation darstellen. Fraglich ist jedoch, ob dadurch ein höheres Arbeitsengagement prognostiziert werden kann.

In einer Studie von Netz (2012) wurden Motive für einen Auslandsaufenthalt untersucht. Individuen, die sich für einen Auslandsaufenthalt entscheiden, wollen es sich dadurch erleichtern, in internationalen Unternehmen $\mathrm{zu}$ agieren, und wollen ihrer Karriere einen Aufschwung geben. Eine Umfrage des Hochschul-Informations-System e. G. im Auftrag des Bundesministeriums für Bildung und Forschung ist bei einer Befragung von Absolvent*innen darüber hinaus zu dem Ergebnis gekommen, dass ein deutlich höherer Anteil Studierender mit einem Auslandsaufenthalt ein Promotionsvorhaben anstrebt. Zudem weisen Personen mit einem Auslandsaufenthalt ein höheres Einkommen durch ihre Tätigkeit in international ausgerichteten Unternehmen auf (Netz 2012). Diese Befunde können als Indikatoren für ein höheres Arbeitsengagement von Personen mit Auslandsaufenthalt gewertet werden. Die vorliegende Studie untersucht daher, inwiefern Personen mit Auslandsaufenthalt ein höheres berufliches Engagement aufweisen als Personen ohne Auslandsaufenthalt.

\subsection{Resilienz gegen Stress}

Stress und dessen Bewältigung sind in der globalisierten Welt zu einem Kernthema geworden. Wie bereits erläutert, gibt es auf dem Arbeitsmarkt mehrere Faktoren, die erhöhte Resilienz gegen Stress erfordern. Zum einen die von Schaarschmidt und Fischer (2008) beschriebenen Anforderungen durch den Anspruch an Mobilität und ständige Verfügbarkeit. Auf der anderen Seite die Anforderung, sich an neue Kulturen schnell und ohne große Reibungen anzupassen. Im Zusammenhang mit Auslandsaufenthalten und der Persönlichkeit wird in verschiedenen Studien vor allem die persönliche Entwicklung des Individuums während des Auslandsaufenthaltes untersucht. Bei den bisher vorhandenen Studien handelt es sich daher größtenteils um Längsschnittstudien. Eine der relevantesten Studien im Zusammenhang mit Auslandsaufenthalten und der Entwicklung der Big-Five-Persönlichkeitsdimensionen ist die Längsschnittstudie von Zimmermann und Neyer (2013). Hier wurden ca. 1000 Probanden vor, während und kurz nach ihrem Auslandsaufenthalt gebeten, einen umfangreichen Persönlichkeitsfragebogen auszufüllen. Es konnten verschiedene Veränderungen in den untersuchten Persönlichkeitsdimensionen nachgewiesen werden. Personen mit Auslandserfahrung zeigten einen Anstieg der Verträglichkeit, Offenheit und eine Verringerung des Neurotizismus. Dabei war vor allem die Dauer der Auslandsaufenthalte entscheidend. Je länger die Auslandsaufenthalte dauerten, desto höher der Anstieg von Verträglichkeit und desto niedriger die Ausprägung von Neurotizismus. Bei Neurotizismus konnte der Abbau jedoch erst nach sechs Monaten im Ausland nachgewiesen werden. Vermutet werden dahinter mögliche Anpassungsschwierigkeiten in der ersten Auslandsphase. Den Ergebnissen von Zimmermann und Neyer (2013) ist zu entnehmen, dass Personen, die länger im Ausland waren weniger negative Emotionen, vor allem weniger Stress erleben. Zimmermann und Neyer (2013) merken an, dass dies möglicherweise auf eine höhere Resilienz gegen potenziell stressige Situationen zurückzuführen ist, die durch eine zunehmende Anzahl überwundener komplexer interkultureller Situationen erworben wird.. Diese Studie untersucht daher, inwiefern Personen, die einen Auslandsaufenthalt absolviert haben, eine höhere Resilienz gegen Stress aufweisen als Personen ohne Auslandsaufenthalt.

\subsection{Interkulturelle und berufliche Kompetenz}

Gelingt es einer Person, stressige Situationen und Akkulturationsprobleme an ihrem neuen Aufenthaltsort zu überwinden, kann es dort im Weiteren nicht nur normal interagieren, sondern hat unter Umständen auch neue Perspektiven und Konstrukte kennengelernt (Thomas 2003). Durch das Leben außerhalb des gewohnten Kultursystems kann das eigene Wissen ausgebaut und die Fähigkeit zum Umgang mit fremden Kulturen verbessert werden. Ob der Umgang mit einer fremden Kultur gelingt, ist dabei von verschiedenen Faktoren abhängig. Einer der stärksten Prädiktoren dafür stellt die kulturelle Intelligenz dar (Deardorff 2006; Genkova und Ringeisen 2015, 2017). Aufgrund der hohen internationalen Vernetzung vieler Branchen, der zunehmenden Internationalisierung sowie Digitalisierung, wird interkulturelle Kompetenz heute als Schlüsselkompetenz verstanden, die auch im Zuge von Personalauswahlverfahren immer stärker in den Fokus von Unternehmen rückt (Kanning 2019). Kulturelle Intelligenz wiederum wird als Teil der emotionalen Intelligenz verstanden und vor allem durch den Austausch von Kulturen untereinander entwickelt (Earley 2002; Earley et al. 2006; Earley und Peterson 2004). Ist eine Person kulturell intelligent, so soll sie Verhaltensweisen und Situationen so interpretieren können, als ob sie dem fremden Kulturkreis angehören würde (Remhof 2015). Diese Person soll folglich befähigt sein, von kultureller Diversität geprägte Situationen $\mathrm{zu}$ begreifen und erfolgreich zu handeln (Ang und Van Dyne 2015; Berry und Ward 
2006). Hohe kulturelle Intelligenz ist zudem geprägt von einem starken persönlichen Interesse an der unbekannten Kultur. Verbindungen zwischen den Kulturen werden eigenständig erkannt und, falls notwendig, wird eine schnelle Anpassungsreaktion auf kulturelle Veränderungen gezeigt (Lee und Templer 2003; Lee und Sukoco 2010).

Moderne Studien betrachten kulturelle Intelligenz als ein mehrdimensionales Konstrukt und differenzieren vier Komponenten: Die metakognitive Komponente beschreibt mentale Prozesse zum Erwerb und Verständnis von kulturellem Wissen (Ang et al. 2015). Die kognitive Komponente bezieht sich auf Wissen über Praktiken, Sitten und Normen unbekannter Kulturen. Die Fähigkeit, Aufmerksamkeit und Energie in das Lernen im Kontext interkultureller Situationen zu investieren, wird der motivationalen Komponente zugeordnet (Van Dyne et al. 2015). Die vierte Komponente ist verhaltensbezogene und umfasst angemessene Verhaltensweisen in interkulturellen Situationen (Ang et al. 2015).

In der Studie von Netz (2012) schätzten Absolvent*innen mit Auslandserfahrung die Bedeutung von kultureller Intelligenz im beruflichen Kontext höher ein als solche ohne Auslandserfahrung. Diese subjektive Einschätzung stimmt mit der beruflichen Entwicklung der Absolvent*innen mit Auslandserfahrung überein. Sie üben vermehrt eine Beschäftigung in Unternehmen mit internationaler Ausrichtung und hoher Reisetätigkeit aus (Netz 2012). Des Weiteren schätzten die Absolvent*innen mit Erfahrungen im Ausland ihre interkulturellen Kompetenzen höher ein als die Personen ohne Auslandserfahrung (Schomburg und Teichler 2006). Es konnte gezeigt werden, dass Sprachkenntnisse und Kenntnisse über fremde Kulturen tatsächlich zunehmen (Dwyner 2004). Die größte Steigerung erfährt kulturelle Intelligenz dadurch, dass Individuen reisen (Erpenbeck 2012; Ward und Kennedy 1999; Van Dyne et al. 2007). Es wird daher angenommen, dass Personen mit Auslandsaufenthalt eine höhere kulturelle Intelligenz aufweisen als Personen ohne.

Wie bereits beschrieben, führten Zimmermann und Neyer (2013) eine große Längsschnittstudie zur Auswirkung von Auslandsaufenthalten auf die Big-Five-Persönlichkeitsmerkmale durch. Trotz der Kritik an der Betrachtung von Persönlichkeitsmerkmalen als veränderbare Konstrukte (siehe dazu detaillierter auch Tracy-Ventura et al. 2016) konnten sie einen Anstieg der Offenheit für neue Erfahrungen und einen Abfall von Neurotizismus nach sechs Monaten Aufenthaltsdauer beobachten. Jedoch konnten die Langzeiteffekte dieser Wirkungen nicht überprüft werden, da die letzte Erhebung jener Untersuchung kurz vor Ende des Auslandsaufenthaltes stattfand und es keine Follow-up Untersuchungen gab.

Um diese Forschungslücke zu schließen, untersucht die vorliegende Studie die Langzeiteffekte eines Auslandsaufenthaltes. Um die zugrundeliegende Forschungsfrage zu beantworten, inwiefern ein Auslandsaufenthalt eine Kompetenzschule darstellt, werden berufsorientierte Konstrukte zur Erfassung der Persönlichkeit verwendet, die an die BigFive angelehnt sind.

\subsection{Hypothesen der vorliegenden Studie}

Das Ziel der vorliegenden Untersuchung ist es aufzuzeigen, wie sich Auslandsaufenthalte auf die interkulturelle Kompetenz und andere berufsbezogene Kompetenzen auswirken. Zudem wurde die Wirkung der Dauer des Aufenthaltes, Sprachkenntnisse, sowie Qualität und Quantität des Kontaktes zu Angehörigen der Gastkultur auf die kulturelle Intelligenz untersucht. Die Analyse dieser unterschiedlichen Facetten ermöglicht, zu einem differenzierteren Bild der Auswirkungen eines Auslandsaufenthaltes zu gelangen.

Diese Auswirkungen können, gemäß dem heutigen Stand des Wissens über Akkulturationsvorgänge, alle Ebenen der menschlichen Psyche betreffen. Diese Arbeit stellt einen Beitrag dazu dar, die langfristigen Folgen eines Auslandsaufenthaltes für die Kompetenz der Befragten zu verstehen, da gerade der praxisbezogene, kompetenzorientierte Aspekt bisher kaum untersucht wurde.

Um diese Forschungsfrage zu beantworten, wurden Personen mit und ohne Auslandserfahrung verglichen. Es wurden folgende Hypothesen formuliert:

H1 Personen mit einem Auslandsaufenthalt weisen ein höheres Arbeitsengagement auf als Personen ohne einen Auslandsaufenthalt, was sich durch höheren Ehrgeiz, höhere subjektive Bedeutsamkeit der Arbeit, höheres Perfektionsstreben und höhere Verausgabungsbereitschaft ausdrückt.

In der zweiten Hypothese wird der Einfluss von Auslandsaufenthalten auf die Resilienz untersucht. Es wird davon ausgegangen, dass Individuen durch die Auslandsaufenthalte, den Abbau von Neurotizismus und die absolvierten Herausforderungen eine höhere Resilienz aufweisen (Schaarschmidt und Fischer 2008).

H2 Personen mit Auslandsaufenthalt weisen eine höhere Resilienz auf. Dies zeigt sich in höherer Distanzierungsfähigkeit, offensiverer Problembewältigung, höherer innerer Ruhe sowie durch eine niedrigere Resignationstendenz bei Misserfolgen.

Es wird angenommen, dass Personen mit Auslandsaufenthalten eine höhere kulturelle Intelligenz aufweisen. Dabei ist zu untersuchen, wie sich die unterschiedlichen Bedingungen des Auslandsaufenthalts auf die kulturelle Intelligenz auswirken. Aus diesem Grund wird der Zusammenhang zwischen der kulturellen Intelligenz und der Dauer des Auslandsaufenthaltes, den Sprachkenntnissen und der Qualität sowie der Quantität der Kontakte zu Angehörigen der Gastkultur untersucht. 
H3 Auslandserfahrungen generell sowie die Dauer des Aufenthaltes, Sprachkenntnisse, Qualität und Quantität des Kontaktes zu Angehörigen der Gastkultur sind Prädiktoren für kulturelle Intelligenz.

In der vorliegenden Studie werden die berufsrelevanten Persönlichkeitsdimensionen im Zusammenhang mit Auslandsaufenthalten untersucht. Es wird vermutet, dass Personen mit Auslandserfahrungen durch die bewältigten Herausforderungen im Ausland höhere Ausprägungen in den zu untersuchenden Persönlichkeitsdimensionen aufweisen. Hier werden berufsbezogene Persönlichkeitsdimensionen untersucht. Diese sind orientiert an dem Konzept der Big-Five, sodass die Befunde mit denen der Studie von Zimmermann und Neyer (2013) verglichen werden können.

H4 Personen mit Auslandserfahrungen zeigen höhere Ausprägungen der berufsbezogenen Persönlichkeitsdispositionen Stabilität, Engagement, Disziplin, soziale Kompetenz, Kooperationsbereitschaft und Dominanz.

\section{Methode}

\subsection{Untersuchungsdurchführung und Messinstrumente}

Zur Testung der Hypothesen wurde eine Querschnittstudie mittels des Online-Erhebungs-Tools LimeSurvey durchgeführt. Die Stichprobe wurde über verschiedene Internetportale, vorrangig Facebook, verbreitet. Die Teilnehmenden wurden darauf hingewiesen, dass die Studie vollkommen anonym und in Übereinstimmung mit geltenden Datenschutzrichtlinien stattfindet. Durch die Teilnahme stimmten sie zu, dass die erhobenen Daten zu ausschließlich wissenschaftlichen Zwecken genutzt werden dürfen. Sie wurden im Weiteren gebeten, ehrlich und spontan zu antworten. Anschließend wurden die folgenden Messinstrumente benutzt: Bei der Abfrage der demografischen Daten und zum Auslandsaufenthalt erfolgte eine Orientierung an dem Fragebogen Socio Cultural Adaption Scale (SCAS) nach Ward und Kennedy (1999), welcher Fragen zum Leben im Ausland beinhaltet. Die Probanden wurden gefragt, ob sie einen Auslandsaufenthalt absolviert hatten. Antworteten sie mit Nein, wurden sie direkt zum nachfolgenden Messinstrument weitergeleitet. Bejahten die Probanden die Frage, folgten acht zusätzliche Items zu den Auslandaufenthalten. Abschließend wurden die Befragten in einer offenen Frage aufgefordert, die Erkenntnisse, welche sie aus dem Auslandsaufenthalt mitnehmen konnten, zu erläutern.

Nach der Angabe der demografischen Daten und gegebenenfalls der Daten zum Auslandsaufenthalt wurden die Befragten zum Fragebogen zur Erfassung von Arbeitsbezogenen Verhaltens- und Erlebensmuster (AVEM) weiter- geleitet. Der AVEM ist ein mehrdimensionales persönlichkeitsdiagnostisches Verfahren und thematisiert die individuellen und persönlichen Ausauseinandersetzungen mit Arbeitsanforderungen. Die Skala, welche von Schaarschmidt und Fischer (2008) entwickelt wurde, basiert auf einer kritischen Auseinandersetzung mit dem Konzept des Typ-AVerhaltens von Friedman und Rosenman (1959). Dieses Konzept wurde um die individuelle Belastbarkeit erweitert. Die Belastbarkeitsmessung nehmen Schaarschmidt und Fischer durch die zu untersuchenden Merkmale Arbeitsengagement, Resilienz und Emotionen vor. Diese Merkmale repräsentieren die personalen und beruflichen Ressourcen des Individuums, welche das Arbeitsverhältnis mitgestalten. In dieser Studie wurde die Kurzversion des AVEM, der AVEM-44, verwendet. Dieser ist insbesondere für die Erhebung mit größeren Stichproben durch die erhöhte Ökonomie geeignet. Der AVEM-44 besteht aus elf Dimensionen, die faktorenanalytisch bestätigt wurden. Die Dimensionen können den übergeordneten Merkmalen Arbeitsengagement (Dimensionen 1-5, Bsp. „Die Arbeit ist für mich der wichtigste Lebensinhalt.“, $\alpha=0,81$ ), Resilienz (Dimensionen 6-8, Bsp. „Mich bringt so leicht nichts aus der Ruhe.", $\alpha=0,76$ ) und Emotionen (Dimensionen 9-11, Bsp. „Im Großen und Ganzen bin ich glücklich und zufrieden.“, $\alpha=0,85$ ) zugeordnet werden.

Es folgte die Erhebung der kulturellen Intelligenz mittels der Cultural Intelligence Scale (CQS) (Van Dyne et al. 2009). Diese misst auf einer siebenstufigen Likert-Skala mit 20 Items vier Subdimensionen, die sich zu einem Gesamtwert verrechnen lassen. Die Dimensionen sind metakognitive kulturelle Intelligenz (Bsp. „Ich prüfe die Richtigkeit meines kulturellen Wissens, indem ich mit Menschen aus verschiedenen Kulturen interagiere.“, $\alpha=0,91$ ), kognitive kulturelle Intelligenz (Bsp. „Ich kenne die kulturellen Werte und religiösen Ansichten von anderen Kulturen.“, $\alpha=0,89$ ), verhaltensbezogene kulturelle Intelligenz (Bsp. „Ich verändere mein Sprachverhalten (z.B. Akzent, Ton), wenn eine interkulturelle Aktion es erfordert.", $\alpha=0,76$ ) und motivationale kulturelle Intelligenz (Bsp. „Ich genieße es in Kulturen zu leben, die mir unbekannt sind.“, $\alpha=0,92$ ).

Abschließend erfolgte die Erhebung des berufsbezogenen Selbstbilds der Persönlichkeit mittels der Kurzform des „Bochumer Inventars zur berufsbezogenen Persönlichkeitsbeschreibung - 6 Faktoren“ (BIP-6F). Der BIP-6F (Hossiep und Krüger 2012) ist als berufsorientiertes Messinstrument der Persönlichkeit an den Big Five angelehnt und misst mittels einer sechsstufigen Likert Skala die Dimensionen Engagement ( $\alpha=0,72)$, Disziplin $(\alpha=0,83)$, Sozialkompetenz $(\alpha=0,76)$, Kooperation $(\alpha=0,93)$, Dominanz $(\alpha=0,84)$ und Stabilität $(\alpha=0,76)$. Gegenüber dem NEO-FFI weist der BIP-6F eine zufriedenstellende inkrementelle Validität auf, da er Lohn und Karriere signifikant besser vorhersagt (Hossiep und Krüger 2012). 


\subsection{Stichprobe}

Der Onlinefragebogen wurde von 367 Personen vollständig bearbeitet; davon waren $71 \%$ weiblich und $29 \%$ männlich. Das Durchschnittsalter der Befragten betrug 30,14 Jahre. Die Probanden waren zwischen 18 und 65 Jahren alt Damit konnten alle Befragten dem berufsfähigen Alter zugeordnet werden. Zudem verfügte die Stichprobe über einen hohen Bildungsgrad. 155 der Teilnehmenden (42,2\% der Stichprobe) gaben an, einen Auslandsaufenthalt absolviert zu haben, 212 hatten keinen. Dabei absolvierten 20,8\% ein Auslandssemester. Der zweithäufigste Grund für einen Auslandsaufenthalt wurde mit einer beruflichen Tätigkeit $(11,2 \%)$ angegeben. Work-and-Travel absolvierten 6,9\% der befragten Personen und 3,3\% verbrachten ein Schuljahr im Ausland. Die durchschnittliche Dauer der Auslandsaufenthalte hat 12,5 Monate betragen. Dabei lag die minimale Aufenthaltsdauer bei einem Monat und die maximale Dauer bei 60 Monaten. 8,6\% der Teilnehmenden gaben an, sechs Monate im Ausland gewesen zu sein, was die häufigste Nennung war.

Die Orte der jeweiligen Auslandsaufenthalte wurden in Kontinente kategorisiert, wobei jedoch Nord- und Südamerika getrennt berichtet werden. In Europa wurden mit 37,87\% die meisten Auslandsaufenthalte absolviert. Dahinter liegen Asien mit 18,18\% und Nordamerika mit $16,66 \%$ der Auslandsaufenthalte. Es gaben 13,64\% an, einen Auslandsaufenthalt in Australien absolviert zu haben und jeweils 7,58\% nannten als Zielländer Südamerika und Südafrika. Fünfzig Prozent der Befragten gaben an, das ihre a-priori-Erwartungen an den Auslandsaufenthalt im GroBen und Ganzen erfüllt worden waren. Nur bei $10,61 \%$ der Probanden hatten die Vorstellungen, die sie vom Gastland gehabt hatten, kaum der Realität entsprochen.

Im Folgenden werden die Ergebnisse erläutert. Tab. 2 stellt die Mittelwerte und Standardabweichungen der Variablen dar.

Tab. 2 Deskriptive Statistik

\begin{tabular}{llllll}
\hline & $N$ & Min & Max & $M$ & $S D$ \\
\hline Dauer (Monate) & 66 & 1,00 & 60,00 & 9,22 & 11,68 \\
Kontaktqualität & 63 & 0 & 4 & 2,29 & 1,07 \\
Kontakthäufigkeit & 66 & 1 & 3 & 2,80 & 0,50 \\
Arbeitsengagement & 152 & 2,13 & 5,00 & 3,40 & 0,50 \\
Resilienz & 152 & 2,25 & 5,00 & 3,03 & 0,33 \\
Kulturelle Intelligenz & 152 & 2,35 & 7,00 & 4,83 & 0,91 \\
Engagement & 152 & 1,63 & 5,13 & 3,54 & 0,76 \\
Disziplin & 152 & 1,88 & 5,88 & 4,35 & 0,72 \\
Sozialkompetenz & 152 & 1,25 & 5,75 & 3,89 & 0,86 \\
Kooperation & 152 & 1,50 & 5,88 & 3,70 & 0,87 \\
Dominanz & 152 & 1,38 & 5,50 & 3,19 & 0,93 \\
Stabilität & 152 & 1,50 & 5,63 & 3,57 & 0,68 \\
\hline
\end{tabular}

\section{Ergebnisse}

\subsection{Hypothese 1 - Arbeitsengagement}

Hypothese 1 besagt, dass es einen signifikanten Unterschied im Arbeitsengagement zwischen den Personen mit versus ohne Auslandsaufenthalt gibt. Dies wurde mittels $\mathrm{t}$-Test überprüft. Der Unterschied ist statistisch signifikant $(t(366)=1,85 ; p=0,03)$. Die Effektstärke nach Pearson liegt bei $r=0,15$ und entspricht damit einem schwachen Effekt (Personen mit Auslandsaufenthalt $M=3,49 ; S D=0,51$; Personen ohne Auslandsaufenthalt: $M=3,33 ; S D=0,49$ ). Damit kann die Hypothese 1 als bestätigt angesehen werden. Personen, die einen Auslandsaufenthalt absolviert haben, weisen demnach ein signifikant höheres Arbeitsengagement auf.

Außerdem wurde untersucht, ob Gruppenunterschiede zwischen Personen mit versus ohne Auslandaufenthalt in den einzelnen Dimensionen von Arbeitsengagement auf Gruppenunterschiede beobachtet werden konnten.

Es wurde mittels t-Test ein signifikanter Unterschied in der Dimension beruflicher Ehrgeiz festgestellt $(t(365)=3,50$; $p=0,005)$. Dieser Effekt kann als mittelstark $(r=0,27)$ bezeichnet werden. Im Durchschnitt zeigen Personen mit Auslandsaufenthalt $(M=3,79 ; S D=0,79)$ einen signifikant höheren beruflichen Ehrgeiz als Personen ohne Auslandsaufenthalt $(M=3,33 ; S D=0,82)$. In der Dimension subjektive Bedeutsamkeit der Arbeit gab es eine nichtsignifikante Tendenz zu einem Unterschied $(t(365)=1,56 ; p=0,06$; $r=0,13)$. In den Dimensionen Perfektionsstreben und Verausgabungsbereitschaft unterschieden sich die Gruppen nicht signifikant voneinander.

\subsection{Hypothese 2 - Resilienz}

Hypothese 2 nimmt an, dass Personen mit Auslandsaufenthalt höhere Ausprägungen in den Dimensionen Resilienz, Distanzierungsfähigkeit, offensive Problembewältigung, innere Ruhe und Ausgeglichenheit sowie Resignationstendenz bei Misserfolgen aufweisen. Es zeigt sich ein signifikanter Unterschied in der Ausprägung der Dimension Resilienz $(t(366)=1,68 ; p=0,04 ; r=0,14)$ (Personen mit Auslandsaufenthalt: $M=3,01 ; S D=0,37$; ohne: $M=2,99$; $S D=0,29)$. Somit wird die Hypothese 2 bestätigt. Für ein detaillierteres Verständnis dieses Effekts wurden die jeweiligen Subskalen von Resilienz untersucht.

In Bezug auf die Distanzierungsfähigkeit gab es einen signifikanten Gruppenunterschied $(t(365)=2,13 ; p=0,02)$. Die Effektstärke nach Pearson liegt bei $r=0,17$ und entspricht einem schwachen Effekt (Probanden mit Auslandsaufenthalt: $M=2,91 ; S D=0,56$; ohne: $M=2,72 ; S D=0,53$ ).

Bei der offensiven Problembewältigung erbrachte der Levene-Test einen Hinweis auf eine mögliche Verletzung 
der Varianzhomogenitätsannahme $(p=0,07)$. Daher wurde ein Welch-Test durchgeführt, der einen signifikanten Unterschied anzeigte $(t(148,64)=2,00 ; p=0,02)$. Die Effektstärke nach Pearson liegt mit $r=0,16$ bei einem schwachen Effekt (Personen mit Auslandsaufenthalt: $M=3,55 ; S D=0,59$; ohne: $M=3,34$; $S D=0,70$ ).

Auf der Dimension innere Ruhe und Ausgeglichenheit wurden die Unterschiede statistisch nicht signifikant $(t(365)=0,56 ; p=0,29 ; r=0,05)$. Auch die Variable niedrige Resignationstendenz bei Misserfolgen weist statistisch keine signifikanten Unterschiede in den Mittelwerten auf $(t(365)=-0,62 ; p=0,27 ; r=0,05)$.

\subsection{Hypothese 3 - Kulturelle Intelligenz}

Zur Überprüfung der Hypothese 3 zur Ausprägung der kulturellen Intelligenz in Abhängigkeit von verschiedenen demografischen Angaben wurden ein t-Test sowie Regressionen berechnet.

Hypothese 3 stellte die Vermutung dar, dass Personen mit einem Auslandsaufenthalt eine höhere Ausprägung der kulturellen Intelligenz aufweisen. Da diese Hypothese gerichtet formuliert ist, wird sie durch einen einseitigen t-Test überprüft. Dabei stellte sich heraus, dass sich die Mittelwerte signifikant unterscheiden $(t(365)=5,16 ; p=0,01)$. Der Effekt ist dabei mittelstark bis stark $(r=0,39)$. Im Durchschnitt weisen Personen mit einem Auslandsaufenthalt einen höheren Mittelwert $(M=5,22 ; S D=0,85)$ bei der kulturellen Intelligenz auf als Personen ohne Auslandsaufenthalt $(M=4,52 ; S D=0,83)$.

Es lässt sich ein schwacher positiver Zusammenhang zwischen der Dauer des Auslandsaufenthaltes und der Ausprägung der kulturellen Intelligenz feststellen $\left(\beta=0,32^{*}\right)$.

Bei der Prüfung des Zusammenhangs zwischen Sprachkenntnissen und kulturelle Intelligenz zeigte sich kein signifikanter Zusammenhang ( $p=0,59)$.

Der Zusammenhang zwischen der Qualität der Kontakte zum Gastland und der kulturellen Intelligenz stellte sich mit $\beta=0,31 *$ als signifikant dar. Somit wird die die Hypothese $3 c$ bestätigt. Es besteht also ein schwacher Zusammenhang zwischen der Qualität der Kontakte zum Gastland und der kulturellen Intelligenz.

Zwischen der Variable Quantität der Kontakte zu Angehörigen der Gastkultur und der kulturellen Intelligenz zeigte sich jedoch kein signifikanter Zusammenhang. Damit konnte Hypothese 3 nur teilweise bestätigt werden.

\subsection{Hypothese 4 - berufliche Leistungsfähigkeit}

Zur Prüfung der vierten Hypothese wurden die Ausprägungen verschiedene Dimensionen der beruflichen Leistungsfähigkeit zwischen den Personengruppen verglichen. Die untersuchten Dimensionen sind Stabilität, Engagement, Dis- ziplin, soziale Kompetenz, Kooperationsbereitschaft sowie Dominanz. Der einseitige t-Test ergab für die Dimension Stabilität keinen signifikanten Unterschied $(t(365)=1,28$; $p=0,10 ; r=0,10)$. In der Dimension Engagement zeigte sich jedoch ein signifikantes Ergebnis $(t \quad(365)=2,94$; $p=0,01)$. Dabei ist das Ergebnis mit $r=0,23$ mittelstark.

Die Mittelwertsunterschiede zu den Dimensionen Disziplin, soziale Kompetenz und Kooperationsbereitschaft sind nicht signifikant.

In Bezug auf die Dominanz unterscheiden sich die Befragten signifikant voneinander $(t(159)=2,58 ; p=0,01)$. Der Effekt liegt bei $r=0,21$ und entspricht damit einem mittelstark Effekt (Personen mit Auslandserfahrung: $M=3,41$; $S D=0,96$; ohne: $M=3,51 ; S D=0,87)$.

Zusammenfassend kann damit auch Hypothese 4 als teilweise bestätigt betrachtet werden.

\section{Diskussion und Limitationen}

Das Ziel der Studie war es, die Auswirkungen von Auslandsaufenthalten auf die berufsbezogenen Kompetenzen Arbeitsengagement (Ehrgeiz, subjektive Bedeutsamkeit der Arbeit, Perfektionsstreben und Verausgabungsbereitschaft), Resilienz (Distanzierungsfähigkeit, offensive Problembewältigung, innere Ruhe sowie niedrige Resignationstendenz), kulturelle Intelligenz und berufliche Leistungsfähigkeit (Stabilität, Engagement, Disziplin, soziale Kompetenz, Kooperationsbereitschaft und Dominanz) mit ihren jeweiligen Subkompetenzen zu untersuchen. Somit war es möglich, die Anforderungen an Internationalität und Flexibilität im Arbeitsleben (Biemann 2009) mit Blick auf Auslandsaufenthalte und deren Auswirkung auf das näher zu untersuchen.

Es wurden Unterschiede zwischen Teilnehmenden mit und ohne Auslandsaufenthalt hinsichtlich mehrerer Variablen aufgedeckt. Die Befragten mit Auslandserfahrung zeigten ein höheres Arbeitsengagement und gaben einen stärkeren beruflichen Ehrgeiz an. Zudem wiesen Personen mit Auslandserfahrung eine höhere Resilienz gegen Stress auf. Darüber hinaus waren sie offensiver in der Problembewältigung und eher in der Lage, sich im Privatleben von der Arbeit zu distanzieren.

Die Ergebnisse zeigen ebenfalls, dass die Befragten, die einen Auslandsaufenthalt absolviert haben, eine höhere Ausprägung der kulturellen Intelligenz aufweisen. Dabei ist zu erwähnen, dass die kulturelle Intelligenz mit der Dauer des Auslandsaufenthaltes und der Qualität der Kontakte zu Einheimischen der Gastkultur zusammenhängt: Je höher die Qualität des Kontaktes zu Angehörigen der Gastkultur und je länger der Aufenthalt, desto höher liegen auch die Werte der kulturellen Intelligenz. 
In Bezug auf die berufliche Leistungsfähigkeit zeigen die Befragten mit Auslandserfahrung ein höheres Engagement sowie eine höhere soziale Kompetenz. Des Weiteren sind Personen mit Auslandsaufenthalten im beruflichen Kontext dominanter.

Da diese Kombination leistungsorientierter Variablen und interkultureller Kompetenz nach Kenntnis der Autoren bisher noch von keinem anderen Forschungsteam untersucht wurde, wird für die Einordnung der Ergebnisse auf Studien zurückgegriffen, die ähnliche Konzepte untersuchten. Die Ergebnisse der vorliegenden Studie stehen im Einklang mit den Befunden der Hochschul-InformationsSystem e. G., dass Personen mit Auslandsaufenthalten beruflich ehrgeiziger sind und zudem ein höheres Arbeitsengagement aufweisen (Netz 2012). Dabei ist der berufliche Ehrgeiz von besonderer Relevanz, da höherer Ehrgeiz zu einem höheren Arbeitsengagement führt.

Auch das Ergebnis der vorliegenden Studie, dass Personen mit Auslandserfahrung eher eine Promotion anstreben und in größeren sowie internationalen Unternehmen beruflich tätig sind, deckt sich mit den Ergebnissen der Hochschul-Informations-System e. G. (Netz 2012).

Die Ergebnisse replizieren ebenfalls den Befund von Zimmermann und Neyer (2013), dass Personen mit Auslandserfahrung eine höhere Resilienz gegen Stress aufweisen und die Mobilität von Studierenden allgemein das Empfinden von Stress reduzieren kann (Andrews et al. 1993). Die Resilienz wirkt sich vor allem auf den Umgang mit Problemen dahingehend aus, dass die Befragten sich offensiver in der Problemlösung sowie distanzierter zu möglichen Stressoren zeigen. Während Zimmermann und Neyer (2013) eine Verminderung von Neurotizismus während der Aufenthalte im Ausland für eine stärkere Resilienz verantwortlich machen, müsste die genaue Natur des Zusammenhangs in nachfolgenden Studien überprüft werden.

Der Anstieg der interkulturellen Kompetenz infolge von Auslandsaufenthalten ist mit der Hypothese von Ward und Kennedy (1999) vereinbar, dass die kulturelle Intelligenz am ehesten durch Erfahrungen in fremden Kulturen erreicht wird. Dabei ist der Anstieg vor allem von der Dauer des Aufenthaltes und der Qualität des Kontaktes zu den Angehörigen der fremden Kultur abhängig. Weniger relevant sind hingegen die Quantität des Kontaktes sowie die Sprachkenntnisse, womit die Ergebnisse von Dwyner (2004) nicht repliziert wurden.

Die Bedingungen aus der Kontakthypothese (Allport 1981) wurden in dieser Studie nicht weiter berücksichtigt, da sie keine theoretisch notwendigen Voraussetzungen oder Rahmenbedingungen für die in dieser Studie untersuchten Zusammenhänge darstellen. Dennoch stehen die Befunde der vorliegenden Befunde in Beziehung zur Kontakthypothese, da Allport (1981) davon ausging, dass eine erhöhte Kontaktintensität das Verständnis für fremde Kulturen an- steigen lässt. Jedoch wurde in der vorliegenden Studie nicht untersucht, ab welcher Dauer ein Anstieg der kulturellen Intelligenz zu verzeichnen ist. Zimmermann und Neyer (2013) bspw. stellten einen Anstieg der Persönlichkeitsdimensionen erst nach einem Auslandsaufenthalt von sechsmonatiger Dauer fest. Diese Differenzierung wurde in der vorliegenden Studie nicht vorgenommen.

Die unterschiedlichen Ausprägungen in den Persönlichkeitsdimensionen zur beruflichen Leistungsfähigkeit können sind ähnlich zu den Ergebnissen von Zimmermann und Neyer (2013). Während Zimmermann und Neyer (2013) einen Anstieg in der Verträglichkeit und Offenheit im Verlauf der Auslandsaufenthalte gefunden haben, zeigte sich in der vorliegen Studie einen Anstieg beim Engagement sowie der Dominanz. Es werden jedoch keine Unterschiede in der sozialen Kompetenz und Kooperationsbereitschaft gemessen, die noch am ehesten zu der Persönlichkeitsdimension Verträglichkeit von Zimmermann und Neyer (2013) passen würde.

Hinsichtlich der Problematik der Veränderbarkeit von Persönlichkeitseigenschaften hat die Kompetenzforschung widerholt Kritik grundsätzlicher Art geerntet, da nach verbreiteten Definitionen Persönlichkeitseigenschaften stabil sein sollten. Tracy-Ventura et al. (2016) zeigten jedoch nachvollziehbar auf, dass einschneidende Lebensereignisse, wie zum Beispiel ein Auslandsaufenthalt zu Änderungen der Persönlichkeit beitragen können (Wolff 2017), womit entweder das Konzept von Persönlichkeitseigenschaften überarbeitet werden sollte, oder aber die Benennung der sich verändernden Variablen modifiziert werden sollte.

Die quantitativen Befunde der vorliegenden Studie decken sich mit qualitativen Beschreibungen zu den Wirkungen der Auslandsaufenthalte, mittels der offenen Frage erhoben wurden: „Was konnten Sie aus den Auslandsaufenthalten mitnehmen?". Diese qualitativen Ergebnisse wurden im Ergebnisteil nicht genannt, da Sie keinen Beitrag zur Testung der Hypothesen liefern, allerdings zur Interpretation der Ergebnisse. Die häufigsten Nennungen in diesem Bereich waren eine Verbesserung der Sprachkenntnisse und die Steigerung interkultureller Kompetenzen mit jeweils 81 Nennungen. Des Weiteren vermuten 66 Personen eine Veränderung der Persönlichkeit und 12 betonen die neuen Kontakte in fremden Kulturen. Damit lassen sich die Ergebnisse auch aus Sicht der Personen mit Auslandserfahrung in Bezug auf die Hypothesen hinterlegen und bestätigen.

\subsection{Limitationen}

Einige der vorliegenden Hypothesen konnten nicht bestätigt werden. Die Konstrukte subjektive Bedeutsamkeit der Arbeit, Perfektionsstreben, Verausgabungsbereitschaft, innere Ruhe und Ausgeglichenheit, Resignationstendenz, Stabilität, Disziplin, soziale Kompetenz und Kooperationsbe- 
reitschaft zeigten keine signifikanten Mittelwertdifferenzen zwischen den Personengruppen mit versus ohne Auslandaufenthalt. Zudem besteht kein Zusammenhang zwischen den Sprachkenntnissen des Gastlandes sowie der Quantität des Kontaktes zu den Angehörigen der Gastkultur und der Ausprägung der kulturellen Intelligenz.

Neben den nicht signifikanten Ergebnissen weist die Erhebung weitere Limitationen auf, wie insbesondere die homogene Stichprobe in Bezug auf den Bildungsgrad und die ungleichen Häufigkeiten der Geschlechter sowie die ungleichen Häufigkeiten der beiden zu untersuchenden Personengruppen mit $u$ ohne Auslandserfahrung. Hier ist jedoch zu beachten, dass auch in der Grundgesamtheit nicht die Hälfte der Personen einen Auslandsaufenthalt absolviert. Daher ist die ungleiche Verteilung in diesem Punkt zu vernachlässigen. Die ungleiche Verteilung in anderen Punkten kann durch die Verbreitungsmethode über soziale Netzwerke und den Bekanntenkreis begründet werden. Zudem handelt es sich mit einem Durchschnittsalter von 30 Jahren um eine sehr junge Stichprobe. Für die Betrachtung von Studienabsolventen und Absolventinnen, sowie Berufseinsteigern ist das Alter jedoch angemessen.

Die Effektstärken der Mittelwertsunterschiede sind überwiegend klein bis mittelgroß. Der mittlere Unterschied zwischen den Personen mit und ohne Auslandserfahrung wird also wahrscheinlich auch in der zugrundeliegenden Grundgesamtheit von Studierenden und Alumnis auftauchen, ist aber im Mittel nicht außerordentlich groß. Dies ist insofern nicht verwunderlich, als dass allein diese Studie bereits verschiedene mögliche Kovariaten identifiziert hat, die möglicherweise Einfluss auf den Kompetenzgewinn nehmen und so den Effekt des Auslandsaufenthaltes an sich reduzieren. Während Wolff (2017) verschiedene mögliche Kovariaten in Studien zu Auslandsaufenthalten diskutiert, würde eine ausführliche Diskussion dessen den Rahmen dieses Beitrags sprengen. Es soll an dieser Stelle lediglich darauf hingewiesen werden, dass auch ein Kompetenzunterschied von mittlerer Größe zu einem deutlich anderen Arbeitsergebnis führen und über Erfolg oder Misserfolg entscheiden kann.

Eine weitere Schwäche weist die Studie im Bereich der Angabe der Auslandsaufenthalte auf. Zwar wurden die Teilnehmenden gebeten, bei der Befragung nur ihren längsten Auslandsaufenthalt anzugeben, jedoch fiel bei den offenen Fragen zu den Orten der Auslandsaufenthalte auf, dass drei Befragte mehrere Auslandsaufenthalte angaben. Daher kann bei der Dauer der Auslandsaufenthalte nicht ausgeschlossen werden, dass eine Verzerrung vorliegt und sich die Befragten auf mehrere Auslandsaufenthalte bezogen haben. In zukünftigen Studies sollten daher eine Mehrfachauswahl und eine Unterscheidung zwischen Orten und der jeweiligen Dauer von Auslandsaufenthalten ermöglicht werden, oder ein anderes Untersuchungsdesign gewählt wer- den, bei dem Vor- und Nachuntersuchungen zu jeweils einem Auslandsaufenthalt stattfinden (Wolff 2017).

Während Wolff (2017) darauf hinweist, dass die kulturelle Distanz zwischen Heimat- und Zielland als möglicher zusätzlicher Prädiktor untersucht werden sollte, bringt diese Variable gleichzeitig Probleme der Operationalisierung mit sich. Da Maßstäbe für die kulturelle Distanz methodisch fragwürdig sind und momentan kein zufriedenstellendes Verfahren vorliegt, wurde in der vorliegenden Studie darauf verzichtet die jeweiligen Länder der Aufenthalte als Indikator für die möglicherweise erlebte kulturelle Distanz zum Heimatland zu berücksichtigen. Die anderen Untersuchungsergebnisse sind davon jedoch nicht betroffen, da hier lediglich der Unterschied zwischen Befragten mit und ohne Auslandserfahrung untersucht wurde.

Bei Persönlichkeitstests und Selbsteinschätzungen besteht zudem grundsätzlich die Gefahr des sozial erwünschten Antwortverhaltens, welches auch in dieser Studie nicht ausgeschlossen werden kann. Zwar wurde mit der Einleitung und der Betonung der Anonymität versucht, diesem Effekt entgegenzuwirken, dennoch kann das sozial erwünschte Antwortverhalten in selbstreflektierenden Einschätzungen nie ausgeschlossen werden.

\subsection{Fazit}

Die Befunde dieser Studie weisen darauf hin, dass es durch Auslandsaufenthalte zu weitreichenden Veränderungen der berufsbezogenen Kompetenzen kommt. Als Implikationen für die Praxis können daher Handlungsempfehlungen abgeleitet werden.

Es zeigte sich, dass sich Personen, die einen Auslandsaufenthalt absolviert haben durch erhöhten beruflichen Ehrgeiz, erhöhtes Engagement und eine stärkere Resilienz im Umgang mit Problemen sowie eine erhöhte Dominanz auszeichnen.

Diese Kompetenzen und die erhöhte Dominanz sind vor allem in hierarchisch höheren Positionen und bei Positionen mit Führungsverantwortung Erfolgsprädiktoren (Kanning 2014). Es erscheint daher vertretbar, dass Unternehmen auch bei Stellenanzeigen für nicht-internationale Tätigkeiten Auslandserfahrung von den Bewerber*innen fordern. Insbesondere bei Führungspositionen sind beruflich ehrgeizige Bewerber*innen und ein gesundes dominantes Verhalten wünschenswert. Die Motive für einen Auslandsaufenthalt sind jedoch im Lebenslauf nicht nachvollziehbar. Daher ist die Eignung dieses Kriteriums für die Bewerber*innenvorauswahl mit einem Fragezeichen zu versehen.

Eine erhöhte offensive Problembewältigung, eine gesunde Distanzierungsfähigkeit sowie eine erhöhte Resilienz können Arbeitnehmer*innen generell helfen, die zunehmenden Beanspruchungen im Arbeitskontext zu bewältigen, unabhängig von dessen internationaler Ausrichtung 
(Scherrmann 2015). Zudem kann durch eine erhöhte Resilienz die Stressbelastung insgesamt reduziert werden. Auslandsaufenthalte können daher ein Indiz für die Belastungsfähigkeit von Bewerber*innen sein.

Eine erhöhte kulturelle Intelligenz kann entscheidend für den erfolgreichen Einsatz von Arbeitnehmer*innen im Ausland sowie bei der internationalen Zusammenarbeit sein (Kanning 2019; Genkova und Ringeisen 2017). Bei der Auswahl von Bewerber*innen für Positionen, die interkulturelle Kontaktsituationen mit sich bringen, können daher vorherige Auslandsaufenthalte ein Indiz für die Eignung sein. Grade für berufliche Aufenthalte im Ausland wird allerdings empfohlen, Angestellte gezielt vorzubereiten, auch wenn diese bereits Auslandserfahrung haben, vor allem, wenn der vorangegangene Aufenthalt nicht in derselben Zielkultur erfolgte (Genkova und Ringeisen 2015, 2017).

Die Ergebnisse der vorliegenden Studie deuten also darauf hin, dass Auslandsaufenthalte tatsächlich als potenzielle Kompetenzschulen zu betrachten sind, welche Empathie, Wissen und Perspektivwechsel fördern können. Dennoch sollten Auslandsaufenthalte nicht global als Kompetenzschule für alle Berufe betrachtet werden. Stellenanforderungen sollten stets differenziert analysiert werden. Allein über Auslandserfahrungen können im Auswahlprozess natürlich nicht alle relevanten Verhaltensweisen von Bewerber*innen vorhergesagt werden.

Für zukünftige Studien bietet es sich an, anhand gröBerer Stichproben und längsschnittlichen Untersuchungen Vorher-, Nachhermessungen die Wirkungen unterschiedlicher Arten von Auslandsaufenthalten und unterschiedlicher Längen besser vergleichbar zu machen, sodass Rückschlüsse auf die genaue Wirkung auf die berufsrelevanten Persönlichkeitsmerkmale möglich werden. Auf dieser empirischen Basis könnten relevante Kriterien in Personalauswahlprozesse zu integriert werden. Aus der Sicht praxis-orientierter Forschung ist darüber hinaus die Notwendigkeit zu betonen, Erfolgsprädiktoren interkultureller Zusammenarbeit im Tätigkeitsspektrum der jeweiligen Position zu identifizieren, und so die Effizienz der Personalauswahl zu steigern (Kanning 2019).

Funding Open Access funding enabled and organized by Projekt DEAL.

Open Access Dieser Artikel wird unter der Creative Commons Namensnennung 4.0 International Lizenz veröffentlicht, welche die Nutzung, Vervielfältigung, Bearbeitung, Verbreitung und Wiedergabe in jeglichem Medium und Format erlaubt, sofern Sie den/die ursprünglichen Autor(en) und die Quelle ordnungsgemäß nennen, einen Link zur Creative Commons Lizenz beifügen und angeben, ob Änderungen vorgenommen wurden.

Die in diesem Artikel enthaltenen Bilder und sonstiges Drittmaterial unterliegen ebenfalls der genannten Creative Commons Lizenz, sofern sich aus der Abbildungslegende nichts anderes ergibt. Sofern das betreffende Material nicht unter der genannten Creative Commons Lizenz steht und die betreffende Handlung nicht nach gesetzlichen Vorschriften erlaubt ist, ist für die oben aufgeführten Weiterverwendungen des Materials die Einwilligung des jeweiligen Rechteinhabers einzuholen.

Weitere Details zur Lizenz entnehmen Sie bitte der Lizenzinformation auf http://creativecommons.org/licenses/by/4.0/deed.de.

\section{Literatur}

Allport, G.W. (1981). The nature of prejudice. Cambridge: AddisonWesley.

Andrews, G., Page, A.C., \& Neilson, M. (1993). Sending your teenagers away: controlled stress decreases neurotic vulnerability. Archives of General Psychiatry, 50, 585-589.

Ang, S., \& Van Dyne, L. (2015). Conceptualization of cultural intelligence: definition, distinctiveness, and nomological network. In Handbook of cultural intelligence (S. 21-33). London: Routledge.

Ang, S., Van Dyne, L., \& Rockstuhl, T. (2015). Cultural intelligence: origins, conceptualization, evolution, and methodological diversity. In M. J. Gelfand, C. Chiu \& Y. Hong (Hrsg.), Handbook of advances in culture and psychology $\mathrm{Bd}$. 5. London: Oxford.

Berry, J.W. (1990). Psychology of acculturation. In J.J. Berman (Hrsg.), Current theory and research in motivation. Nebraska Symposium on Motivation, 1989: Cross-cultural perspectives. (Bd. 37, S. 201-234). Lincoln: University of Nebraska Press.

Berry, J.W., \& Ward, C. (2006). Commentary on "redefining interactions across cultures and organizations". Group and Organization Management, 31(1), 64-77.

Biemann, T. (2009). Die Bedeutung internationaler Erfahrungen für den Karriereerfolg von Führungskräften. Zeitschrift für Personalforschung, 23(4), 336-356.

Bologna-Erklärung (1999). Der Europäische Hochschulraum. Gemeinsame Erklärung der Europäischen Bildungsminister. http://www. bmbf.de/pubRD/bologna_deu.pdf. Zugegriffen: 6. Juli 2017.

Deardorff, D. K. (2006). Identification and assessment of intercultural competence as a student outcome of internationalization. Journal of Studies in international Education, 10(3), 241-266.

Dick, R. V., Wagner, U., Adams, C., \& Petzels, T. (2014). Einstellungen zur Akkulturation. In A. Glöckner-Rist (Hrsg.), Zusammenstellung sozialwissenschaftlicher Items und Skalen. Bonn: GESIS.

Dwyner, M. M. (2004). More is better: the impact of study abroad program duration. The Interdisciplinary Journal of Study Abroad, 10, $51-163$.

Earley, P.C. (2002). Redefining interactions across cultures and organizations: moving forward with cultural intelligence. Research in Organizational Behavior, 24, 271-299.

Earley, P.C., \& Peterson, R. S. (2004). The elusive cultural chameleon: cultural intel-ligence as a new approach to intercultural training for the global manager. Academy of Management Learning and Education, 3(1), 100-115.

Earley, P.C., Ang, S., \& Tan, J.S. (2006). CQ: Developing cultural intelligence at work. Stanford: Stanford University Press.

Erpenbeck, J. (2012). Siegeszug trotz Kritik: Warum Kompetenzdenken notwendig ist. Wirtschaftspsychologie Aktuell, 19, 19-22.

Europäische Kommission (2010). Jugend in Bewegung. Eine Initiative zur Freisetzung des Potenzials junger Menschen, um in der Europäischen Union intelligentes, nachhaltiges und integratives Wachstum zu erzielen. Luxemburg: Amt für Veröffentlichungen der Europäischen Union.

Friedman, M., \& Rosenman, R. (1959). Association of specific overt behaviour pattern with blood and cardiovascular findings. Journal of the American Medical Association, 169, 1286-1296.

Genkova, P., \& Ringeisen, T. (2015). Handbuch Diversity Kompetenz. Wiesbaden: Springer.

Genkova, P., \& Ringeisen, T. (2017). Gegenstandsbereiche. Bd. 2. Wiesbaden: Springer. 
Hammer, M. R. (2015). The Developmental paradigm for intercultural competence research. International journal of intercultural relations, 48(1), 12-13.

Helfrich, H. (2013). Kulturvergleichende Psychologie. Wiesbaden: Springer.

Heublein, U., Hutzsch, C., \& Lörz, M. (2008). Auslandsmobilität deutscher Studierender: Ausmaß, Motive und Gründe des Desinteresses. Zeitschrift für Bildung und Erziehung, 61(4), 437-450.

Hossiep, R. \& Krüger, C. (2012). Bochumer Inventar zur berufsbezogenen Persönlichkeitsbeschreibung - 6 Faktoren (BIP-6F). Göttingen: Hogrefe.

Kanning, U. P. (2014). Inventar zur Messung sozialer Kompetenzen in Selbst- und Fremdbild (ISK-360). Göttingen: Hogrefe.

Kanning, U.P. (Hrsg.). (2019). Lehrbuch der Personalpsychologie. Göttingen: Hogrefe.

Lee, C.H., \& Templer, K. J. (2003). Cultural intelligence assessment and measurement. In P.C. Earley \& S. Ang (Hrsg.), Cultural intelligence: individual interactions across cultures (S. 185-208). Palo Alto: Stanford University Press.

Lee, L. Y., \& Sukoco, B. M. (2010). The effects of cultural intelligence on expatriate performance: the moderating effects of international experience. The International Journal of Human Resource Management, 21(7), 963-981.

Netz, N. (2012). Exkurs: Studienbezogene Auslandsmobilität und Berufsverbleib von Hochschulabsolventinnen. In M. Grotheer, S. Isleib, N. Netz \& K. Briedis (Hrsg.), Hoch qualifiziert und gefragt. Hannover: HIS.

Remhof, S. (2015). Stand der Forschung und theoretischer Hintergrund. In Absicht zur Arbeit im Ausland (S. 11-25). Wiesbaden: Springer.

Repo, H., Vahlberg, T., Salminen, L., Papadopoulos, I., \& Leino-Kilpi, H. (2017). The cultural competence of graduating nursing students. Journal of transcultural nursing, 28(1), 98-107.

Schaarschmidt, U., \& Fischer, A. W. (2008). AVEM - Arbeitsbezogenes Verhaltens- und Erlebensmuster. London: Pearson.

Schartner, A. (2016). The effect of study abroad on intercultural competence: a longitudinal case study of international postgraduate students at a British university. Journal of multilingual and multicultural development, 37(4), 402-418.

Scherrmann, U. (2015). Stress und Burnout in Organisationen - Ein Praxishandbuch für Führungskräfte, Personalentwickler und Berater. Berlin Heidelberg: Springer.

Schomburg, H., \& Teichler, U. (2006). Higher education and graduate employment in europe: results from graduates surveys from twelve countries. Higher education dynamics. Dordrecht: Springer.

Silbereisen, R. K., \& Schmitt-Rodermund, E. (1993). Entwicklungsorientierungen jugendlicher Aussiedler: Unterschiede je nach Auslandsdauer und Akkulturationserfahrungen. Zeitschrift für Sozialisationsforschung und Erziehungssoziologie, 13, 341-364.

Thomas, A. (Hrsg.). (2003). Kulturvergleichende Psychologie (2. Aufl.). Göttingen: Hogrefe.

Tracy-Ventura, N., Dewaele, J. M., Köylü, Z., \& McManus, K. (2016). Personality changes after the 'year abroad'?: a mixed-methods study. Study Abroad Research in Second Language Acquisition and International Education, 1(1), 107-127. https://doi.org/10. 1075/sar.1.1.05tra.

Van Dyne, L., Ang, S., \& Nielsen, T. M. (2007). Cultural intelligence. In S. Clegg \& J. Bailey (Hrsg.), International encyclopaedia of organization studies (S. 345-350). Thousand Oaks: SAGE.

Van Dyne, L., Ang, S., \& Koh, C. (2009). Cultural intelligence: Measurement and scale development. In M. A. Moodian (Hrsg.), Contemporary leadership and intercultural competence: exploring the cross-cultural dynamics within organizations (S. 233-254). Thousand Oaks: SAGE.
Van Dyne, L., Ang, S., \& Koh, C. (2015). Development and validation of the CQS: the cultural intelligence scale. In Handbook of cultural intelligence (S. 34-56). London: Routledge.

Ward, C. A., \& Kennedy, A. (1999). The measurement of sociocultural adaptation. International Journal of Intercultural Relations, 23, 659-677.

Ward, C., Bochner, S., \& Furnham, A. (2001). The psychology of cultural shock. London: Routledge.

Wolff, F. (2017). Interkulturelle Kompetenz durch Auslandsaufenthalte. Wiesbaden: Springer. https://doi.org/10.1007/9783658163921.

Zick, A. (2010). Psychologie der Akkulturation - Neufassung eines Forschungsbereichs. Wiesbaden: Springer Gabler.

Zimmermann, J., \& Neyer, F. J. (2013). Do we become a different person when hitting the road? Personality development of sojourners. Journal of personality and social psychology, 105(3), 515.

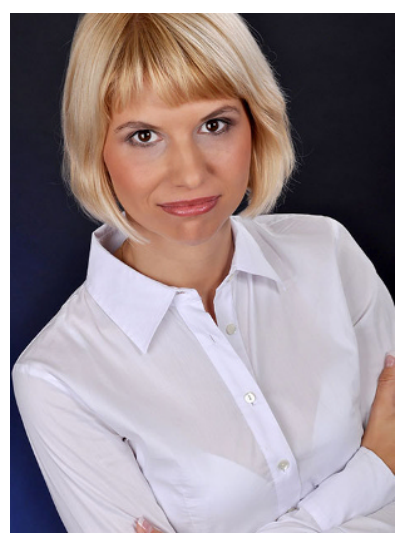

Prof. Dr. phil. habil. Petia Genkova ist Professorin für Wirtschaftspsychologie an der Fakultät für Wirtschafts- und Sozialwissenschaften an der Hochschule Osnabrïck und leitet mehrere Forschungsprojekte, die der vertiefenden Forschung der Interkulturellen Kompetenz, Interkulturellen Kommunikation und Zusammenarbeit sowie dem Erleben und der Gesundheit und Zufriedenheit von Personen mit Migrationshintergrund als auch dem Thema Diversity dienen. Zudem ist sie Vorstandsmitglied beim Deutschen Akademikerinnen Bund sowie Mitglied in verschiedenen Fachgesellschaften und -verbänden, z. B. Delegierte im Frauenrat NRW, International Association of Cross-Cultural Psychology, Vorsitzende der Sektion Politische Psychologie und Sprecherin des Gleichstellungsausschusses beim Berufsverband Deutscher Psychologinnen und Psychologen (BDP). Zudem fungiert Frau Prof. Genkova als Gutachterin beim Bundesministerium für Bildung und Forschung, Wissenschaftsrat, Schweizerischen Nationalfonds und Internationalen Journals und ist Trägerin des HöffmannWissenschaftspreises für Interkulturelle Kompetenz für Lebenswerk.

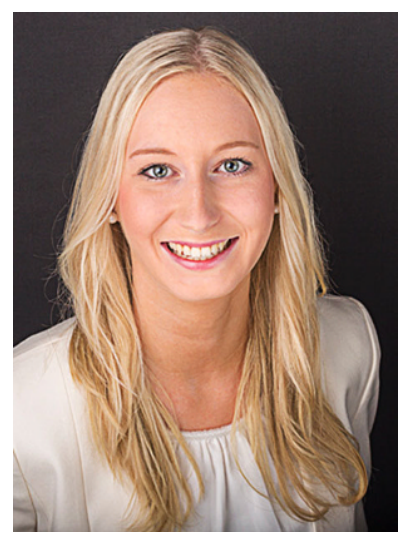

Lisa Kruse studierte Wirtschaftspsychologie an der Hochschule Osnabrück und Arbeits- und Organisationspsychologie an der Bergischen Universität Wuppertal und ist seitdem als Referentin für Recruiting tätig. 